\title{
Fractional Supersymmetry As A Matrix Model
}

\author{
Ilham Benkaddour and El Hassane Saidi \\ UFR-High Energy Physics, Department of Physics, Faculty of Sciences, \\ Rabat University, Av. Ibn Battouta B.P. 1014, Morocco. \\ H-saidi@fsr.ac.ma \\ November 1, 2018
}

\begin{abstract}
Using parafermionic field theoretical methods, the fundamentals of $2 \mathrm{~d}$ fractional supersymmetry $\mathbf{Q}^{K}=P$ are set up. Known difficulties induced by methods based on the $U_{q}(s l(2))$ quantum group representations and non commutative geometry are overpassed in the parafermionic approach. Moreover we find that fractional supersymmetric algebras are naturally realized as matrix models. The $K=3$ case is studied in details. Links between $2 \mathrm{~d}\left(\frac{1}{3}, 0\right)$ and $\left(\left(\frac{1}{3}^{2}\right), 0\right)$ fractional supersymmetries and $\mathrm{N}=2$ $\mathrm{U}(1)$ and $\mathrm{N}=4 \mathrm{su}(2)$ standard supersymmetries respectively are exhibited. Field theoretical models describing the self couplings of the matter multiplets $\left(0^{2},\left(\frac{1}{3}\right)^{2},\left(\frac{2}{3}\right)^{2}\right)$ and $\left(0^{4},\left(\frac{1}{3}\right)^{4},\left(\frac{2}{3}\right)^{4}\right)$ are given.
\end{abstract}

PACS numbers: 0220, 1110K, 1125H, 1130P

Published in Class.Quantum Grav. 16(1999)1793-1804 


\section{Introduction}

In the few last years, there has been attempts to develop a superspace formulation of 2d QFT that is invariant under fractional supersymmetry (FSS) defined by: $Q^{k}=$ $P, \quad \bar{Q}^{k}=\bar{P}, \quad K>2$, together with relations involving both $\mathrm{Q}$ and $\bar{Q}$. In these Eqs $P=P_{-1}$ and $\bar{P}=P_{+1}$ are the two heterotic components of the 2 d energy momentum vector operator $P_{m} ; \quad Q \equiv Q_{-1 / k}$ and $\bar{Q} \equiv Q_{1 / k}$ are the basic generator of FSS which carry a $2 \mathrm{~d}$ spin $s=1 / k$. Most of the studies of this special class of $2 \mathrm{~d}$ massive QFT are essentially based on methods of $U_{q}(s l(2))$ quantum group representations and non commutative geometry $[1,2]$. This approach however met many difficulties and has lead to partial results only. Among these difficulties we quote the two following ones associated with the heterotic $\mathrm{K}=3$ case $\left(Q^{3}=P\right)$. The first one deals with the computation of the two points correlation function $<\Psi_{-1 / 3}\left(z_{1}\right), \Psi_{-2 / 3}\left(z_{2}\right)>$ of the two partners $\Psi_{-1 / 3}(z)$ and $\Psi_{-2 / 3}(z)$ of the bosonic field $\varphi(z)$ of the scalar representation $\left(\varphi, \Psi_{-1 / 3}, \Psi_{-2 / 3}\right)$ of the $\left(Q^{3}=P\right)$ algebra. From the view point of the $2 \mathrm{~d}$ conformal field theory, one expects from the braiding feature $z_{12} \Psi_{-1 / 3}\left(z_{1}\right) \Psi_{-2 / 3}\left(z_{2}\right)=z_{21} \Psi_{-2 / 3}\left(z_{2}\right) \Psi_{-1 / 3}\left(z_{1}\right)$, that the field operators $\Psi_{-1 / 3}$ and $\Psi_{-2 / 3}$ should anticommute. This result however is not fulfiled in the approach based on the $U_{q}(s l(2))$ quantum groups and non commutative geometry where the $\Psi_{-1 / 3}$ and $\Psi_{-2 / 3}$ fields obey a generalised commutation rule [3] leading to a two point correlation function $<\Psi_{-1 / 3}\left(z_{1}\right), \Psi_{-2 / 3}\left(z_{2}\right)>_{U_{q}(s l(2))}$ which violate locality.

The second thing we want to quote concerns the construction of the generalised superspace and superfields. In this context, one uses generalised Grassmann variables $\theta_{1}=\theta_{1 / 3}$ and $\theta_{2}=\theta_{-1 / 3}$ satisfying a third order nilpotency condition $\theta_{ \pm 1 / 3}^{3}=0$, together with $\theta_{ \pm 1 / 3}^{2} \neq 0$ and $\theta_{1} \theta_{2}=\omega \theta_{2} \theta_{1}$, where $\omega$ is a $\mathrm{C}$-number such that $\omega^{3}=1$. The

condition $\theta_{ \pm 1 / 3}^{3}=0$, which generalizes the usual condition of Grassmann variables of spin $1 / 2$, is necessary in order to describe off shell representations of the fractional supersymmetric algebra (FSA) in terms of superfields $\Phi(z, \theta, \bar{z}, \bar{\theta})$. In this language, FSS is generated by translations along the $\theta$ and $\bar{\theta}$ directions, i.e. $\theta \rightarrow \theta+\varepsilon$ and $\bar{\theta} \rightarrow \bar{\theta}+\bar{\varepsilon}$, where $\varepsilon$ and $\bar{\varepsilon}$ are generalized Grassmann variables of same nature as $\theta$ and $\bar{\theta}$. Until now things are quite similar to the superspace formulation of ordinary supersymmetry associated with $\mathrm{K}=2$. However there are weak points in the construction of the generalised superspaces for $K>2$. One of these weakness deals with the treatemenent of $\theta^{2}, \bar{\theta}^{2}$ and the $\varepsilon$ and $\bar{\varepsilon}$ parameters. In trying to establish a given relation by following two equivalent paths, we get different results. On one hand, $\theta(\bar{\theta})$ should commute with itself as required by the identity $(1-x) \theta^{2}=0$, which implies that either $\theta^{2}=0$ and $x \neq 0$ or $x=1$ and $\theta^{2} \neq 0$. On the other hand $\theta(\bar{\theta})$ and $\varepsilon(\bar{\varepsilon})$ are required to satisfy generalized commutation rules type $\theta \varepsilon=\omega \varepsilon \theta(\bar{\theta} \bar{\varepsilon}=\omega \bar{\varepsilon} \bar{\theta}) ; \omega^{3}=1$. This is a contradiction since $\theta(\theta+\varepsilon) \neq \omega(\theta+\varepsilon) \theta$. This difficulty may be also viewed 
from the generalised commutation rule $\theta \eta=\omega \eta \theta$. Taking the limit $\eta=\theta$, one obtains $\theta^{2}=\omega \theta^{2}$ which implies that $\theta^{2}$ should be zero.

The aim of this paper is to set up the basis of $2 \mathrm{~d}$ fractional supersymmetric QFT by using parafermionic field theoretical methods. These methods, which were considered recently in [4], seems to be the right language to develop a local fractional supersymmetric QFT. This believe is also supported by the fact that after all fractional supersymmetry is nothing but a residual symmetry of massive perturbations of parafermionic critical models. From this point of view; the generators of fractional supersymmetry are just remanant constants of motion surviving after deformations of parafermionic conformal invariance. Thinking of $2 \mathrm{~d}$ fractional supersymmetry as a finite dimensional subsymmetry of the $Z_{K}$ conformal invariance [5] for instance, one discovers that all the known difficulties of the abovementioned approach disappear. Moreover we find that fractional supersymmetric algebras are generated by more than one charge operator and can be described in a natural way as a matrix model. The charge operators $Q_{-x}, \quad x=0,1 / k, \ldots$, form altogether a $K \times K$ matrix operator allowing to define the fractional supersymmetric algebra as:

$$
\operatorname{tr} Q^{k}=P_{-1}
$$

together with other relations. For details see Eqs (5); (8) and (17).

This paper is organized as follows. In section 2, we study the matrix realisations of $2 \mathrm{~d}$ supersymmetry. In section 3, we work out the links between fractional supersymmetry and parafermions. Matrix realisations of fractional supersymmetry are analysed in sections 4 and 5 . In section 6 , we describe brefly the superfield theory of the matter couplings of $\left(\frac{1}{3}, \frac{1}{3}\right)$ and $\left(\left(\frac{1}{3}\right)^{2},\left(\frac{1}{3}\right)^{2}\right)$ fractional superalgebras. In section 7 , we present discussions and give our conclusion.

\section{$1.12 \mathrm{~d}$ supersymmetry as a matrix model}

In this paragraph we consider the $2 \mathrm{~d}$ heterotic supersymmetric algebras respectively defined as:

$$
\begin{array}{llr}
\left\{Q_{-1 / 2}, Q_{-1 / 2}\right\} & = & P_{-1} \\
{\left[P_{-1}, Q_{-1 / 2}\right]} & = & 0
\end{array}
$$

and

$$
\begin{array}{llr}
\left\{Q_{-1 / 2}^{+}, Q_{-1 / 2}^{-}\right\} & = & P_{-1} \\
\left\{Q_{-1 / 2}^{ \pm}, Q_{-1 / 2}^{ \pm}\right\} & = & 0 \\
{\left[P_{-1}, Q_{-1 / 2}^{ \pm}\right]} & = & 0
\end{array}
$$

and shows that they can be represented as $2 \times 2$ matrix models. A generalisation to higher dimensional spaces of these representations turns out to offer a natural framework to study fractional supersymmetry. It shows moreover that methods of standard 
supersymmetry can be also used to deal with exotic supersymmetries.

To start consider the following off diagonal symmetric $2 \times 2$ matrix $\mathbf{Q}$ whose entries are given by the $2 \mathrm{~d}\left(\frac{1}{2}, 0\right)$ supersymmetric generator $Q_{-1 / 2}$ :

$$
\mathbf{Q}=\left[\begin{array}{cc}
0 & Q_{-1 / 2} \\
Q_{-1 / 2} & 0
\end{array}\right]
$$

This matrix operator acts on the $2 \mathrm{~d}$ space of quantum states $|B\rangle=B(0)|0\rangle$ and $|F\rangle=F(0)|0\rangle$ where the letters $\mathrm{B}$ and $\mathrm{F}$ stand for Bose and Fermi fields. Taking the square product of $\mathrm{Eq}(4)$, it is not difficult to see that the $\left(\frac{1}{2}, 0\right)$ supersymmetric algebra (2) may be defined as:

$$
\begin{array}{lll}
\operatorname{tr}\left[\mathbf{Q}^{2}\right] & =P_{-1} \\
\operatorname{tr}[\mathbf{Q}] & =0,
\end{array}
$$

where the suffix tr means the usual matrix trace operation. There are several remarkable features of the matrix definition Eqs (4-5) of the $2 \mathrm{~d}\left(\frac{1}{2}, 0\right)$ supersymmetric algebra; some of them are manifest at the level of the $K \times K=2 \times 2$ matrix model, others are hidden and emerge for $K \geq 3$ representations. For details see sections 3 and 4 .

Moreover combining the usual constraints of $2 \mathrm{~d}$ supersymmetry, in particular hermiticity of the energy momentum vector $P_{-1}, P_{-1}^{+}=P_{-1}$ with the features of the matrix model (5), one sees that the realisation of $\mathbf{Q}, \mathrm{Eq}(4)$, is not the unique one. Indeed, decomposing $\mathbf{Q}$ as $\mathbf{Q}=\mathbf{Q}^{+}+\mathbf{Q}^{-}$

$$
\mathbf{Q}^{+}=\left[\begin{array}{cc}
0 & Q_{-x}^{+} \\
0 & 0
\end{array}\right] ; \quad \mathbf{Q}^{-}=\left[\begin{array}{cc}
0 & 0 \\
Q_{-y}^{-} & 0,
\end{array}\right]
$$

one sees that the spins $\mathrm{x}$ and y carried by the $Q^{ \pm}$charge operators are related as

$$
x+y=1
$$

as required by the first relation of $\mathrm{Eq}(5)$. Abstraction done from $\mathrm{Eq}(2)$ and (3), there are infinitely many solutions of Eqs $(6-7)$; we shall first consider the two solutions $x=y=\frac{1}{2}$ and $\mathrm{x}=0$ and $\mathrm{y}=+1$ related with the leading zero mode operators of the NS and Ramond sectors of $2 \mathrm{~d}$ superconformal invariance. Later on we shall explore the interesting cases $x=\frac{1}{k}$ and $y=\frac{(k-1)}{k}, k=3,4, \ldots$, and their link with $Z_{K}$ parafermionic invariance.Now using Eqs (6) instead of Eq (4) one finds that the algebra Eqs (5) reads in general as:

$$
\begin{array}{lll}
\operatorname{tr}\left[\mathbf{Q}^{+} \mathbf{Q}^{-}\right]=P_{-1} & & \\
\operatorname{tr}\left[\mathbf{Q}^{+} \mathbf{Q}^{+}\right]=\operatorname{tr}\left[\mathbf{Q}^{-} \mathbf{Q}^{-}\right] & = & 0 \\
\operatorname{tr}\left[\mathbf{Q}^{+}\right]=\operatorname{tr}\left[\mathbf{Q}^{-}\right] & = & 0 .
\end{array}
$$

Eqs (8) has a U(1) automorphism symmetry which breaks down to $Z_{2}$ in the case of Eqs (5), this is why we shall refer to Eqs (5) and Eqs $(8)$ as $2 d\left(\frac{1}{2}, 0\right)$ and $\left(\left(\frac{1}{2}\right)^{2}, 0\right)$ 
supersymmetric algebras respectively. Moreover introducing the generators of the gl $(2)$ Lie algebra, we have the following $2 \times 2$ matrix representations:

(i). the NS like superalgebra.

In this case the supersymmetric $2 \times 2$ matrix generators $Q^{ \pm}$read as:

$$
\begin{aligned}
& \mathrm{Q}^{+}=Q_{-1 / 2} E_{12}, \\
& \mathrm{Q}^{-}=Q_{-1 / 2} E_{21},
\end{aligned}
$$

for $\left(\frac{1}{2}, 0\right)$ supesymmetry and

$$
\begin{aligned}
& \mathrm{Q}^{+}=Q_{-1 / 2}^{+} E_{12}, \\
& \mathbf{Q}^{-}=Q_{-1 / 2}^{-} E_{21}
\end{aligned}
$$

for $\left(\left(\frac{1}{2}\right)^{2}, 0\right)$ supersymmetry.

(ii). The Ramond like algebra.

Here the matrix generators are realized as:

$$
\begin{aligned}
& \mathbf{Q}^{+}=Q_{0} E_{12}, \\
& \mathbf{Q}^{-}=Q_{-1} E_{21}
\end{aligned}
$$

for the hermitian case

$$
\begin{aligned}
& \mathrm{Q}^{+}=Q_{0}^{+} E_{12}, \\
& \mathbf{Q}^{-}=Q_{-1}^{-} E_{21},
\end{aligned}
$$

for the complex one. Note that compatibility between the usual hermiticity $(+)$ of the energy momentum vector $P_{-1}$ and the adjoint conjugation $(+)$ of the $Q^{ \pm}$matrix generators requires the following identifications:

$$
\begin{aligned}
& {\left[Q_{-x}^{ \pm}\right]^{+}=Q_{-1+x}^{\mp}} \\
& {\left[Q_{-x}\right]^{+}=Q_{-1+x}}
\end{aligned}
$$

Eqs (11) show that $Q_{-1 / 2}$ is self adjoint whereas $Q_{0}$ and $Q_{-1}$ are interchanged under the $(+)$ conjugation. However knowing that $Q_{0}$ should satisfy a $2 \mathrm{~d}$ Clifford algebra $\left\{Q_{0}, Q_{0}\right\}=1$, and using the current modes of the Ramond superconformal algebra [6], one may set $2 Q_{-1}=\left\{Q_{0}, P_{-1}\right\}=2 Q_{0} P_{-1}$. This identification reduces the number of generators of the algebra Eq (11). Likewise, one may also set $Q_{-1}^{-}=Q_{0}^{-} P_{-1}$ where $Q_{0}^{-}$ together with $Q_{0}^{+}$satisfy

$$
\begin{aligned}
& \left\{Q_{0}^{+}, Q_{0}^{-}\right\}=1 \\
& \left\{Q^{ \pm}, Q^{ \pm}\right\}=0
\end{aligned}
$$

and where $Q_{0}=Q_{0}^{+}+Q_{0}^{-}$. Before going ahead note that the $2 \mathrm{~d}$ NS like superalgebra generated by $Q_{-1 / 2}\left(Q_{-1 / 2}^{ \pm}\right)$exchanges bosons $\mathrm{B}$ and fermions $\mathrm{F}$ whereas the Ramond like one generated by $Q_{0}\left(Q_{0}^{ \pm}\right)$preserves the statistics. In the $2 \mathrm{~d}$ quantum field language, 
the Ramond like algebra acts on the field doublet $\left(\Phi_{1 / 2}(z), \Phi_{-1 / 2}(z)\right), \quad Q_{0}^{ \pm}, \Phi_{ \pm 1 / 2}=$ $0 ; \quad Q_{0}^{ \pm} \Phi_{\mp 1 / 2}=\Phi_{ \pm 1 / 2}$, as follows:

$$
\Phi_{ \pm 1 / 2} \longrightarrow{ }_{Q_{0}^{\mp}} \quad \Phi_{\mp 1 / 2} \longrightarrow Q_{0}^{\mp} \quad \partial_{z} \Phi_{ \pm 1 / 2} .
$$

Note, moreover that the NS and R like algebras can be realized by help of Grassman variables $\theta_{ \pm 1 / 2}^{ \pm}$and $\theta_{0}^{ \pm}$as:

$$
\begin{array}{lll}
Q_{-1 / 2}^{ \pm} & = & \frac{\partial}{\partial \theta_{1 / 2}^{\mp}}+\frac{1}{2} \theta_{1 / 2}^{ \pm} \partial_{-1} \\
Q_{0}^{ \pm} & = & \frac{\partial}{\partial \theta_{0}^{\mp}}+\frac{1}{2} \theta_{0}^{ \pm} .
\end{array}
$$

Similar realizations may be also written down for the hermitian charge operators $Q_{-1 / 2}$ and $Q_{-1 / 2}$. In what follows, we want to extend the above matrix formulation to the case $\mathrm{K}=3$. At first sight this should be possible if one succeeds to relate fractional supersymmetry to an infinite dimensional fractional superconformal invariance in the same manner as usual $2 \mathrm{~d}$ supersymmetry is related to the superconformal invariances. To that purpose we start by establishing the link between fractional supersymmetry and the $Z_{K}$ parafermionic invariance. Then we study its matrix realisation.

\section{$2 \quad$ Fractional supersymmetry and parafermions}

Here we want to show that from the point of view of $2 \mathrm{~d} Z_{K}$ parafermionic invariance, the definition of fractional supersymmetry, say $\left(Q_{-1 / k}\right)^{k}=P, K>2$, is just a formal one. The right way to define it is as in Eqs (1). The latter show that FSS is in fact generated by many charge operators which we denote as $Q_{-x}, x=0,1 / k, \ldots$..More precisely FSS is generated by a $k \times k$ matrix $\mathbf{Q}$ whose entries are the charge operators $Q_{-x}$ carrying various values of fractional spins. For the $k=3$ case we are interested in here, there are in total three pairs of charge operators $Q_{-x}^{ \pm}, x=0,1 / 3,2 / 3$, together with $P_{-1}$. Altogether, these operators generate the formal cubic nonlinear algebra $Q^{3}=P_{-1}$ which as we shall see, is correctly defined as

$$
\begin{array}{llr}
\operatorname{tr}\left[\mathbf{Q}^{+} \mathbf{Q}^{-}\right] & = & P_{-1} \\
\operatorname{tr}\left[\mathbf{Q}^{+2}\right]=\operatorname{tr}\left[\mathbf{Q}^{-2}\right] & = & 0 \\
\operatorname{tr}\left[\mathbf{Q}^{+}\right]=\operatorname{tr}\left[\mathbf{Q}^{-}\right] & = & 0 .
\end{array}
$$

In equation $(12) Q^{ \pm}$are $3 \times 3$ matrices given by:

$$
\mathbf{Q}^{+}=\left[\begin{array}{ccc}
0 & 0 & Q_{-1 / 3}^{-} \\
Q_{-2 / 3}^{-} & 0 & 0 \\
0 & Q_{-1}^{-} & 0
\end{array}\right] ; \quad \mathbf{Q}^{-}=\left[\begin{array}{ccc}
0 & Q_{-1 / 3}^{+} & 0 \\
0 & 0 & Q_{0}^{+} \\
Q_{-2 / 3}^{+} & 0 & 0,
\end{array}\right]
$$


Note by the way that here also $Q_{-x}^{+}$and $Q_{-y}^{-}$obey Eqs $(13)$ and $Q_{-1}^{-}=Q_{0}^{-} P_{-1}$. A way to derive Eqs (17-18) is to consider the $Z_{3}$ parafermionic invariance of Zamolodchikov and Fateev (ZF) [5] given by :

$$
\begin{aligned}
& \Psi^{ \pm}\left(z_{1}\right) \Psi^{ \pm}\left(z_{2}\right) \approx-z_{12}^{-2 / 3} \Psi^{ \pm}\left(z_{2}\right) \\
& \Psi^{+}\left(z_{1}\right) \Psi^{-}\left(z_{2}\right) \approx-z_{12}^{-4 / 3}\left[1+5 / 3 z_{12}^{2} T\left(z_{2}\right)\right] \\
& T\left(z_{1}\right) \Psi^{ \pm}\left(z_{2}\right) \approx \frac{2 / 3}{z_{12}^{2}} \Psi^{ \pm}\left(z_{2}\right)+\frac{1}{z_{12}} \partial_{z} \Psi^{ \pm}\left(z_{2}\right) \\
& T\left(z_{1}\right) T\left(z_{2}\right)=\frac{k-1}{k+2} z_{12}^{-4}+2 z_{12}^{-2} T\left(z_{2}\right)+z_{12}^{-1} T\left(z_{2}\right) .
\end{aligned}
$$

In these Eqs $T(z)$ is the usual energy momentum current, $\Psi^{ \pm}(z)\left(\left[\Psi^{ \pm}(z)\right]^{+}=\Psi^{\mp}(z)\right)$ are the ZF parafermionic currents of spin ${ }^{2}$. The algebra (19) has three parafermionic highest weight representations $(\mathrm{PHWR})\left[\Phi_{q}^{q}\right] ; q=0,1,2$. namely the identity family $I=\left[\Phi_{0}^{0}\right]$ of highest weight $h_{0}=0$ and two degenerate families $\left[\Phi_{1}^{1}\right]$ and $\left[\Phi_{2}^{2}\right]$ of weights $h_{1}=h_{2}=\frac{1}{15}$. Each one of these PHWR $\left[\Phi_{q}^{q}\right]$ is reducible into three Virasoro HWR $\left(\Phi_{q}^{p}\right), p=q, p=q+\bmod 6$. For more details on the representation theory of Eqs (18) see [5-7]. All we need in the achievement of this study is the algebra Eqs (19), the identities

$$
\begin{aligned}
& \Psi^{ \pm} \times \Phi_{q}^{p}=\Phi_{q}^{p \pm 2} \\
& \Phi_{q}^{p \pm 6}=\Phi_{q}^{p}
\end{aligned}
$$

as well as the following braiding property of the conformal field operators $\Phi_{1}\left(z_{1}\right)$ and $\Phi_{2}\left(z_{2}\right)$ :

$$
z_{12}^{\Delta} \Phi_{1}\left(z_{1}\right) \Phi_{2}\left(z_{2}\right)=z_{21}^{\Delta} \Phi_{2}\left(z_{2}\right) \Phi_{1}\left(z_{1}\right)=\Phi(z),
$$

where $\Delta=\Delta_{1}+\Delta_{2}+\Delta_{3}, \Delta_{i}, i=1,2,3$, are the weights of the $\Phi_{i}$ 's. Note that Eq (20-a) is a formal Eq which should be understood as:

$$
\Psi^{ \pm}\left(z_{1}\right) \Phi_{q}^{p}=\sum z_{12}^{n-1 \mp p / 3} Q_{-n \pm(p \pm 1) / 3}^{ \pm} \Phi_{q}^{p}\left(z_{2}\right)
$$

where $Q_{-n \pm(p \pm 1) / 3}^{ \pm}$are the mode operators of $\Psi^{ \pm}$which in turn may be defined as

$$
Q_{-n \pm(p \pm 1) / 3}^{ \pm}\left|\Phi_{q}^{p}\right\rangle=\oint d z z^{n \pm p / 3} \Psi^{ \pm}(z) \Phi_{q}^{p}(0)|0\rangle
$$

In addition to Eqs (20) which predicts the existence of three doublets of the charge operators $Q_{-x}^{ \pm}, x=0,1 / 3,2 / 3$, one also has the braiding feature (21) playing a crucial role in the building of FSS. For example the deformation parameter $\omega$ (often denoted by $\mathrm{q}$ in the literature ) of quantum groups and non commutative geometry dealing with fractional supersymmetry should be related with the braiding property of the conformal field blocks $\Phi_{1}\left(z_{1}\right)$ and $\Phi_{2}\left(z_{2}\right)$. Eq (21) tells us that the parameter $\omega$ is equal to $\exp ( \pm i \pi \Delta)$. For $\Delta_{3}=0$ for instance, $\omega$ reduces to $\exp \left( \pm i\left[\pi\left(\Delta_{1}+\Delta_{2}\right)\right]\right)$, so that for $\Delta_{1}+\Delta_{2}=1$, the field operators $\Phi_{1}$ and $\Phi_{2}$ anticommute and then should be 
treated as fermions. Choosing $\Delta_{1}=1 / 3$ and $\Delta_{2}=2 / 3$ for example, the two point function $<\Phi_{1 / 3}\left(z_{1}, \Phi_{2 / 3}\left(z_{2}>\right.\right.$ should be equal to $-<\Phi_{2 / 3}\left(z_{2}, \Phi_{1 / 3}\left(z_{1}>=1 / z_{12}\right.\right.$. This result, derived from parafermionic conformal field methods, solves the difficulty of refs [3] according to which the two point function $<\Phi_{1 / 3}\left(z_{1}, \Phi_{2 / 3}\left(z_{2}>_{U_{q}(s l(2)}\right.\right.$ computed in a model of fractional supersymmetry based on quantum groups and non commutative geometry methods; i.e:

$$
<\Phi_{1 / 3}\left(z_{1}, \Phi_{2 / 3}\left(z_{2}>_{U_{q}(s l(2)}=e^{2 i \pi / 3}<\Phi_{2 / 3}\left(z_{2}, \Phi_{1 / 3}\left(z_{1}>_{U_{q}(s l(2)}=\frac{1}{z_{12}} .\right.\right.\right.\right.
$$

Eq (24) shows that the model based on the $U_{q}(\operatorname{sl}(2)$ methods is non local. In summary local $2 \mathrm{~d}$ field theoretical realisation of fractional supersymmetry cannot be generated by only one charge operator. The number of generators may be obtained by analysing the mode operators $Q_{-n \pm(p \pm 1) / 3}^{ \pm}$, n integer. The $Q_{-n \pm(p \pm 1) / 3}^{ \pm}$'s depend on the p charge of the conformal representation $\left|\Phi_{q}^{p}\right\rangle$ on which they act. For $\mathrm{q}=0$ for example, the non vanishing actions of $Q_{-x}^{ \pm}, x=0,1 / 3,2 / 3$ on the leading states $|s, p\rangle$ of spin $\mathrm{s}, 0 \leq s \leq 1$ and charge $\mathrm{p}$ read as:

$$
\begin{array}{ll}
Q_{-2 / 3}^{ \pm}|0,0\rangle & =\left|\frac{2}{3}, 0\right\rangle \\
Q_{0}^{+}\left|\frac{2}{3},+2\right\rangle & =\left|\frac{2}{3},-2\right\rangle \\
Q_{0}^{-}\left|\frac{2}{3},-2\right\rangle & =\left|\frac{2}{3},+2\right\rangle \\
Q_{-1 / 3}^{+}\left|\frac{2}{3},-2\right\rangle & =|1,0\rangle \\
Q_{-1 / 3}^{-}\left|\frac{2}{3},+2\right\rangle & =|1,0\rangle .
\end{array}
$$

From these Eqs and Eqs (22-23), one sees that $Q_{-1 / 3}^{ \pm}$and $Q_{0}^{ \pm}$cannot act directly on the state $|0,0\rangle$ similarly $Q_{-2 / 3}^{ \pm}$cannot operate directly on $\left|\frac{2}{3}, \pm 2\right\rangle$. This feature gives another indication that FSS should be generated by more than one Q operator as it is currently used in the literature based on quantum groups and non commutative geometry approaches. Recall that the first indication we have mentionned in the introduction of this paper refers to the inconsistences induced by the introduction of the generalized Grassmann variable $\theta$ satisfying a higher order nilpotency condition $\theta^{k}=0, k>2$ with $\theta^{k-1} \neq 0$ and where the problem of locality rised above is just one of the manifestation of the limit of the methods used. In the approach based on parafermionic conformal field theorical techniques we are considering here, these problems are overpassed. Locality is restored since all fields carrying fractional spins obey an anticommuting statistics and the higher order Grassmann nilpotency necessary for the description of off shell representations of FSS is ensured by the presence of more than one charge operator. Having discussed the link between FSS and parafermions, we turn now to study its relation with matrix theory. 


\section{Fractional supersymmetry as a matrix model}

Starting from Eqs (25) and denoting by $\Pi_{r}, r=0, \pm 1$, the projectors along the states $|s, p\rangle=|i\rangle(p=2 i)$ and by $E_{i j}=|i\rangle \otimes|j\rangle$ the generators of the gl(3) Lie algebra rotating the state $|i\rangle$ into the state $|j\rangle, i, j=0, \pm 1$, (the indices \pm refer to the two fractional supersymmetric partners of the bosonic state indexed by $\mathrm{i}=0$.), one sees that the component $P_{-1}$ of the energy momentum vector reads in terms of the $Q_{-x}^{ \pm}$'s, $x=$ $0,1 / 3,2 / 3$ and the projectors as:

$$
\begin{aligned}
& P_{-1} \approx t r \mathbf{P} \\
& \mathbf{P}=Q_{-1 / 3}^{+} Q_{0}^{+} Q_{-2 / 3}^{+} \Pi_{0}+Q_{-2 / 3}^{+} Q_{-1 / 3}^{+} Q_{0}^{+} \Pi_{1}+Q_{0}^{+} Q_{-2 / 3}^{+} Q_{-1 / 3}^{+} \Pi_{-1} .
\end{aligned}
$$

Note that a similar relation to this Eq using $Q_{-x}^{-}$instead of $Q_{-x}^{+}$is also valid . Moreover using Eqs (25), one many rewrite Eqs (26) and its hermetic conjugate in the following form.

$2 P=\left[Q_{-1 / 3}^{+} Q_{-2 / 3}^{-}+Q_{-1 / 3}^{-} Q_{-2 / 3}^{+}\right] \Pi_{0}+\left[Q_{-2 / 3}^{+} Q_{-1 / 3}^{-}+Q_{-1}^{-} Q_{0}^{+}\right] \Pi_{1}+\left[Q_{-2 / 3}^{-} Q_{-1 / 3}^{+}+Q_{-}^{+} Q_{0}^{-}\right] \Pi_{-1}$.

Comparing Eqs (27) and (26), one finds the following constraint relations:

$$
\begin{array}{lll}
Q_{-1 / 3}^{-} & =Q_{-1 / 3}^{+} Q_{0}^{+} \\
Q_{-1 / 3}^{+} & =Q_{-1 / 3}^{-} Q_{0}^{-} \\
Q_{-2 / 3}^{-} & =Q_{0}^{+} Q_{-2 / 3}^{+} \\
Q_{-2 / 3}^{+} & =Q_{0}^{-} Q_{-2 / 3}^{-} \\
Q_{-1}^{-} & =Q_{-2 / 3}^{+} Q_{-1 / 3}^{+} \\
Q_{-1}^{+} & =Q_{-2 / 3}^{-} Q_{-1 / 3}^{-} .
\end{array}
$$

Eqs (28) and consequently Eqs (26-27) may be satisfied identically by introducing the following $3 \times 3$ matrix operator $\mathbf{Q}=\mathbf{Q}^{+}+\mathbf{Q}^{-}$whose entries are $Q_{-x}^{ \pm}$'s, $x=0,1 / 3,2 / 3$ are the generators of FSS. This matrix representation is in agreement with the ZF parafermionic invariance property (22-23) and the constraint Eqs (28). Using the gl(3) generators $E_{i j}=|i\rangle \otimes|j\rangle, i, j=0, \pm 1$, one may write down the two following matrix realisations for the $Q_{-x}^{ \pm}$'s.

(i) $\left(\frac{1}{3}, 0\right)$ Real FSS: This algebra, to which we shall refer hereafter to as $2 \mathrm{~d}\left(\frac{1}{3}, 0\right)$ FSS, is the analogue of Eqs (2) and (5). The matrix representation of the $Q_{-x}^{ \pm}$'s reads as:

$$
\begin{array}{llr}
Q_{-2 / 3}^{+} & = & Q_{-2 / 3} E_{1,0} \\
Q_{-2 / 3}^{-} & = & Q_{-2 / 3} E_{-1,0} \\
Q_{0}^{+} & = & Q_{0} E_{-1,1} \\
Q_{0}^{-} & = & Q_{0} E_{1,-1} \\
Q_{-1 / 3}^{+} & = & Q_{-1 / 3} E_{0,-1} \\
Q_{-1 / 3}^{-} & = & Q_{-1 / 3} E_{0,1}
\end{array}
$$


The charge carried by the $Q_{-x}^{ \pm}$of Eqs (29) is the $Z_{3}$ charge of the automorphism symmetry of the matrix operator equation.

$$
\left(E^{3}\right)_{i i}=\Pi_{i} ; \quad i=0, \pm 1 .
$$

In the orthonormal basis $\{|i\rangle, i=i=0, \pm 1\}$, the matrix representation of the $Q_{-x}^{ \pm}$'s reads as:

$$
\mathbf{Q}^{+}=\left[\begin{array}{ccc}
0 & Q_{-2 / 3} & 0 \\
0 & 0 & Q_{0} \\
Q_{-1 / 3} & 0 & 0
\end{array}\right] ; \quad \mathbf{Q}^{-}=\left[\begin{array}{ccc}
0 & 0 & Q_{-2 / 3} \\
Q_{-1 / 3} & 0 & 0 \\
0 & Q_{-1} & 0,
\end{array}\right]
$$

Using Eqs (31) it is not difficult to check that the following relation hold:

$$
\begin{aligned}
& 2 \mathbf{P}=\mathbf{Q}^{+} \mathbf{Q}^{-}+\mathbf{Q}^{-} \mathbf{Q}^{+} \\
& \mathbf{Q}^{-}=\mathbf{Q}^{+} \mathbf{Q}^{+} \\
& \mathbf{Q}^{-2}=\mathbf{P} \cdot \mathbf{Q}^{+} .
\end{aligned}
$$

Taking the traces of both sides of these matrix Eqs, one discovers the algebra Eqs (17) which reads in terms of $Q_{-1 / 3}$ and $Q_{-2 / 3}$ as:

$$
\begin{aligned}
& P_{-1}=Q_{-1 / 3} Q_{-2 / 3}+Q_{-2 / 3} Q_{-1 / 3} \\
& 0=\left\{Q_{-1 / 3}, Q_{-1 / 3}\right\}=\left\{Q_{-2 / 3}, Q_{-2 / 3}\right\} .
\end{aligned}
$$

Note that $\mathrm{Eq}(33)$ was expected from the constraint Eq (7). It was suggested in ref [ 8] as a linearized form of the non linear operator equation $\left[Q_{-1 / 3}\right]^{3}=P_{-1}$. The relation between Eq (33) and $2 \mathrm{~d}\left(\left(\frac{1}{2}^{2}\right), 0\right)$ supersymmetry suspected in [8] will be considered latter on.

(ii) The $\left(\left(\frac{1^{2}}{3}\right), 0\right)$ FSA: This is a complex solution for which the matrix representation of the $Q_{-x}^{ \pm}$'s reads as.

$$
\mathbf{Q}^{+}=\left[\begin{array}{ccc}
0 & Q_{-2 / 3}^{+} & 0 \\
0 & 0 & Q_{0}^{+} \\
Q_{-1 / 3}^{+} & 0 & 0
\end{array}\right] ; \quad \mathbf{Q}^{-}=\left[\begin{array}{ccc}
0 & 0 & Q_{-2 / 3}^{-} \\
Q_{-1 / 3}^{-} & 0 & 0 \\
0 & Q_{-1}^{-} & 0 .
\end{array}\right]
$$

Here also the charges carried by the $Q_{-x}^{ \pm}$are $Z_{3}$ charges. Similar calculations as for the $\left(\frac{1}{3}, 0\right)$ algebra show that Eqs (32) are again fulfiled for the representation (34). Using Eqs (34) and solving Eqs (32), we find the following relations :

$$
\begin{aligned}
& 2 P_{-1}=\left\{Q_{-2 / 3}^{+}, Q_{-1 / 3}^{-}\right\}+\left\{Q_{-1 / 3}^{+}, Q_{-2 / 3}^{-}\right\} \\
& 0=\left\{Q_{-1 / 3}^{ \pm}, Q_{-1 / 3}^{ \pm}\right\}=\left\{Q_{-2 / 3}^{ \pm}, Q_{-2 / 3}^{ \pm}\right\} \\
& Q_{-1 / 3}^{-}=Q_{-1 / 3}^{+} Q_{0}^{+} ; \quad Q_{-1 / 3}^{+}=Q_{0}^{-} Q_{-1 / 3}^{-} \\
& Q_{-2 / 3}^{-}=Q_{0}^{+} Q_{-2 / 3}^{+} ; \quad Q_{-2 / 3}^{+}=Q_{-2 / 3}^{-} Q_{0}^{-}
\end{aligned}
$$


Eqs $(35)$ define the $\left(\left(\frac{1}{3}\right)^{2}, 0\right)$ fractional supersymmetrc algebra . It is generated by two $Z_{3}$ doublets of anticommuting charges operators $Q_{-1 / 3}^{ \pm}$and $Q_{-2 / 3}^{ \pm}$. The components of each doublet are related to each another by the $Q_{0}^{ \pm}$'s as shown on Eqs (35-c-d). Note that the algebra (35) is stable under the three following conjugations:(a) $\left(Q_{-x}^{ \pm}\right)^{*}=Q_{-x}^{\mp}$; (b) $\left(Q_{-x}^{ \pm}\right)^{+}=Q_{-1+x}^{\mp}$ and $(\mathrm{c})\left(Q_{-x}^{ \pm}\right)^{+*}=Q_{-1+x}^{ \pm}$suggesting a link with $2 \mathrm{~d} \mathrm{~N}=\left(\left(\frac{1}{2}\right)^{4}, 0\right) \operatorname{su}(2)$ supersymmetry formulated in harmonic superspace [9]. In what follows we shall explore this relation in order to use it for the construction of off shell representations of the $2 \mathrm{~d}\left(\left(\frac{1}{3}\right)^{2}, 0\right)$ FSS.

\section{More on the algebra equations (35)}

Because of the periodicity $(p \equiv p \pm 6$ ) of the representations of the ZF parafermionic algebra (19) which allow to identify the field operators $\Phi^{ \pm}$as $\Phi^{ \pm} \equiv \Phi^{ \pm 2}$ and $\Phi^{ \pm \pm} \equiv \Phi^{ \pm 4}$ ; see Eq (20-b) one may rewrite the charge operators $Q_{0}^{+}, Q_{0}^{-}$as $Q_{0}^{--}, Q_{0}^{++}$, respectively. Moreover denoting by $Q_{0}^{0}$ the commutator of the $Q_{0}^{+}$and $Q_{0}^{-}$charge operators, one sees from Eqs (29) that $Q_{0}^{0}$ and $Q_{0}^{ \pm}$generate altogether an $\operatorname{su}(2)$ algebra.

$$
\begin{aligned}
& {\left[Q_{0}^{-}, Q_{0}^{+}\right]=Q_{0}^{0},} \\
& {\left[Q_{0}^{0}, Q_{0}^{ \pm}\right]=\mp Q_{0}^{ \pm},}
\end{aligned}
$$

acting on the $Q_{-x}^{ \pm}, x=\frac{1}{3}, \frac{1}{3}$ charges as:

$$
\begin{aligned}
& {\left[Q_{0}^{0}, Q_{-1 / 3}^{ \pm}\right]= \pm Q_{-1 / 3}^{ \pm}} \\
& {\left[Q_{0}^{0}, Q_{-2 / 3}^{ \pm}\right]= \pm Q_{-2 / 3}^{ \pm}} \\
& {\left[Q_{0}^{ \pm}, Q_{-1 / 3}^{ \pm}\right]=\mp Q_{-1 / 3}^{\mp}} \\
& {\left[Q_{0}^{ \pm}, Q_{-2 / 3}^{ \pm}\right]= \pm Q_{-2 / 3}^{\mp}} \\
& {\left[Q_{0}^{-}, Q_{-x}^{+}\right]=0} \\
& {\left[Q_{0}^{+}, Q_{-x}^{-}\right]=0,}
\end{aligned}
$$

Eqs (36) correspond just to the zero mode subalgebra of the level 3 of the $s u_{3}(2)$ Kac Moody symmetry. The latter is known to be homomorphic to the $Z_{3}$ parafermionic invariance (19) [10]. Now using the identification $Q_{0}^{\mp} \equiv Q_{0}^{ \pm \pm}$and the $Z_{3}$ periodicity $q \equiv \operatorname{qmod}(3)$, one may rewrite the algebra (36) as

$$
\begin{aligned}
& {\left[Q_{0}^{++}, Q_{0}^{--}\right]=Q_{0}^{0}, \quad(a)} \\
& {\left[Q_{0}^{0}, Q_{0}^{ \pm \pm}\right]= \pm Q_{0}^{0} .}
\end{aligned}
$$


Substituting $Q_{0}^{\mp}$ by $Q_{0}^{ \pm \pm}$in Eqs (37); one gets the following relations which looks like the coresponding ones in $2 \mathrm{~d} \mathrm{~N}=4 \mathrm{su}(2)$ supersymmetry [9]:

$$
\begin{aligned}
& {\left[Q_{0}^{++}, Q_{-x}^{+}\right]=\left[Q_{0}^{--}, Q_{-x}^{-}\right]=0,} \\
& {\left[Q_{0}^{++}, Q_{-1 / 3}^{-}\right]=a Q_{-1 / 3}^{+}} \\
& {\left[Q_{0}^{--}, Q_{-2 / 3}^{+}\right]=a Q_{-2 / 3}^{-}} \\
& {\left[Q_{0}^{0}, Q_{-1 / 3}^{ \pm}\right]= \pm a Q_{-1 / 3}^{ \pm}} \\
& {\left[Q_{0}^{--}, Q_{-1 / 3}^{+}\right]=b Q_{-1 / 3}^{-}} \\
& {\left[Q_{0}^{++}, Q_{-2 / 3}^{-}\right]=b Q_{-2 / 3}^{+}} \\
& {\left[Q_{0}^{0}, Q_{-2 / 3}^{ \pm}\right]= \pm a Q_{-2 / 3}^{ \pm},}
\end{aligned}
$$

where $a=-b=1$. Recall that in $2 \mathrm{~d}\left(\left(\frac{1}{3}\right)^{2}, 0\right) \mathrm{su}(2)$ supersymmetry the coefficients a and $\mathrm{b}$ are equal to one, $\mathrm{a}=\mathrm{b}=1$, and the analogue of Eqs $(35-\mathrm{a}-\mathrm{b})$ read as:

$$
\begin{aligned}
& 2 P_{-1}=\left\{Q_{-1 / 2}^{+}, \bar{Q}_{-1 / 2}^{-}\right\}-\left\{Q_{-1 / 2}^{-}, \bar{Q}_{-1 / 2}^{+}\right\} \\
& 0=\left\{Q_{-1 / 2}^{ \pm}, Q_{-1 / 2}^{ \pm}\right\}=\left\{\bar{Q}_{-1 / 2}^{ \pm}, \bar{Q}_{-1 / 2}^{ \pm}\right\}
\end{aligned}
$$

Observe by the way that apart from the spin of the charge operators, Eq (38-a) differs from $\mathrm{Eq}(35-\mathrm{a})$ by the presence of the minus sign which is required by invariance under the $\mathrm{su}(2)$ automorphism group of the $2 \mathrm{~d} \mathrm{~N}=4 \mathrm{su}(2)$ supersymmetric algebra. Nevertheless the similarity between Eqs (35) and (37-38) allows us to build an off shell superspace formulation of $2 \mathrm{~d}\left(\left(\frac{1}{3}\right)^{2}, 0\right)$ supersymmetry by mimiking the harmonic superspace formalism [11].

\section{Superfield theory.}

We start first by describing the superfield theory of the $2 \mathrm{~d}\left(\frac{1}{3}, \frac{1}{3}\right)$, fractional supersymmetry Eq (33) especially the matter couplings of the on shell scalar representation $\left(\varphi, \Psi_{ \pm 1 / 3}, \Psi_{ \pm 2 / 3}\right)$. Using the formal analogy between Eqs (33) and those of $2 \mathrm{~d} N=2$ $\mathrm{U}(1)$ supersymmetry, namely

$$
\begin{array}{lll}
Q_{\mp 1 / 3} & \longrightarrow & Q_{\mp 1 / 2}^{+} \\
\theta_{ \pm 1 / 3} & \longrightarrow & \theta_{ \pm 1 / 2}^{+} \\
Q_{\mp 2 / 3} & \longrightarrow & Q_{\mp 1 / 2}^{-} \\
\theta_{ \pm 2 / 3} & \longrightarrow & \theta_{ \pm 1 / 2}^{-} \\
\varphi & \longrightarrow & \varphi(y) \\
\Psi_{\mp 1 / 3} & \longrightarrow & \Psi_{\mp 1 / 2}^{+} \\
\varphi & \longrightarrow & \varphi(y) \\
\Psi_{\mp 2 / 3} & \longrightarrow & \Psi_{\mp 1 / 2}^{-},
\end{array}
$$

and following the same lines used in the building of $2 \mathrm{~d} N=2 \mathrm{U}(1)$ supersymmetric matter couplings [12], one sees that the superfield action $S[\Phi, \bar{\Phi}]$ invariant under the 
$2 \mathrm{~d}\left(\frac{1}{3}, \frac{1}{3}\right)$ fractional supersymmetry reads as:

$$
S[\Phi, \bar{\Phi}]=\int d^{2} z d \theta_{1 / 3} d \theta_{-1 / 3} d \theta_{2 / 3} d \theta_{-2 / 3} K[\Phi, \bar{\Phi}]
$$

In this Eq, K is the Kahler potential depending on the chiral superfields $\Phi$ and $\bar{\Phi}$ given by :

$$
\begin{aligned}
& \Phi=\varphi+\theta_{1 / 3} \Psi_{-1 / 3}+\theta_{-1 / 3} \Psi_{1 / 3}+\theta_{1 / 3} \theta_{-1 / 3} F \\
& \bar{\Phi}=\bar{\varphi}+\theta_{2 / 3} \Psi_{-2 / 3}+\theta_{-2 / 3} \Psi_{2 / 3}+\theta_{2 / 3} \theta_{-2 / 3} F
\end{aligned}
$$

$\Phi$ and $\bar{\Phi}$ describe a complex scalar representation of the algebra (33); each bosonic degree of freedom has two partners of spin $\frac{1}{3}$ and $\frac{2}{3}$. Note that using Eq (42) and the matter superfield representation Eq (43); one gets the right two point free correlation functions; i.e: $<\Psi_{-1 / 3}\left(z_{1}\right) \Psi_{-2 / 3}\left(z_{2}\right)>$ and $<\Psi_{-1 / 3}\left(z_{1}\right) \Psi_{-1 / 3}\left(z_{2}\right)>=<\Psi_{-2 / 3}\left(z_{1}\right) \Psi_{-2 / 3}\left(z_{2}\right)>=$ 0 . Concerning the $\left(\left(\frac{1}{3}\right)^{2}, 0\right)$ fractional supersymmetric algebra (35) generated by the four generators $Q_{-1 / 3}^{ \pm}$and $Q_{-2 / 3}^{ \pm}$, one may use here also the similarity with $2 \mathrm{~d} N=4$ extended supersymmetry. Thus extending Eqs (35) as:

$$
\begin{aligned}
& P_{-1}=\left\{D_{-2 / 3}^{+}, D_{-1 / 3}^{-}\right\}=-\left\{D_{-1 / 3}^{+}, D_{-2 / 3}^{-}\right\} \\
& 0=\left\{D_{-x}^{ \pm}, D_{-y}^{ \pm}\right\}
\end{aligned}
$$

together with

$$
\begin{aligned}
& {\left[D^{++}, D_{-x}^{+}\right]=\left[D^{++}, D_{-x}^{-}\right]=0 ; \quad x=\frac{1}{3}, \frac{2}{3}} \\
& {\left[D^{++}, D_{-x}^{-}\right]=D_{-x}^{+}} \\
& {\left[D^{++}, D^{--}\right]=D^{0}} \\
& {\left[D^{0}, D_{-x}^{ \pm}\right]= \pm D_{-x}^{ \pm},}
\end{aligned}
$$

and using the Grassman variables $\theta_{1 / 3}^{ \pm}$and $\theta_{2 / 3}^{ \pm}$of spin $\frac{1}{3}$ and $\frac{2}{3}$ respectively, but still satisfying $\theta_{x}^{ \pm 2}=0$ one can build a superspace realisation of Eqs (44-45) and consequently a $2 \mathrm{~d}$ quantum superfield theory. A remarkable realisation of the algebra (44-45), stable under the combined conjugation $+*$ and using the covariant superderivatives $D_{x}^{ \pm}$and $D_{x}^{ \pm \pm}, D_{0}^{0}$ instead of the $Q_{-x}^{ \pm}$generators is given by:

$$
\begin{aligned}
& D_{-1 / 3}^{+}=-\frac{\partial}{\partial \theta_{-1 / 3}^{-}} \\
& D_{-2 / 3}^{+}=-\frac{\partial}{\partial \theta_{-2 / 3}^{-}} \\
& D_{-2 / 3}^{-}=\frac{\partial}{\partial \theta_{2 / 3}^{+}}-\theta_{1 / 3}^{-} P_{-1} \\
& D_{-1 / 3}^{-}=\frac{\partial}{\partial \theta_{1 / 3}^{+}}-\theta_{2 / 3}^{-} P_{-1} \\
& P_{-1}=\frac{\partial}{\partial y}
\end{aligned}
$$

where $y=z-\frac{1}{2}\left(\theta_{1 / 3}^{-} \theta_{2 / 3}^{+}+\theta_{1 / 3}^{+} \theta_{2 / 3}^{-}\right)$and where

$$
\begin{aligned}
& D^{++}=\left[u^{+i} \frac{\partial}{\partial u^{-i}}-\theta_{1 / 3}^{+} \theta_{2 / 3}^{+} P_{-1}\right] \\
& D^{--}=\left[u^{-i} \frac{\partial}{\partial u^{+i}}-\theta_{1 / 3}^{-} \theta_{2 / 3}^{-} P_{-1}\right] \\
& D^{0}=\left[D^{++}, D^{--}\right] .
\end{aligned}
$$


In this realisation $u_{i}^{ \pm}$are the well known harmonic variable satisfying $u^{+i} u_{i}^{-}=1$ and $u^{ \pm i} u_{i}^{ \pm}=0$. Using the realisation (46-47), the on shell matter multiplet $\left(0^{4},\left(\frac{1}{3}\right)^{4},\left(\frac{2}{3}\right)^{4}\right)$ of the fractional supersymmetric algebra (44-45) is given by a hermitean superfield satisfying the analytic conditions

$$
D_{-1 / 3}^{+} \Phi=D_{-2 / 3}^{+} \Phi=0
$$

and the equation of motion

$$
D^{++2} \Phi=0 .
$$

The $\theta_{x}^{+}$expanssion of the superfield $\Phi$, stable under the combined conjugation $(+*)$ reads as:

$$
\Phi=\varphi^{i j} u_{i}^{+} u_{j}^{-}+\theta_{1 / 3}^{+} \Psi_{-1 / 3}^{i} u_{i}^{-}+\theta_{2 / 3}^{+} \Psi_{-2 / 3}^{i} u_{i}^{-}+\theta_{1 / 3}^{+} \theta_{2 / 3}^{+} F^{i j} u_{i}^{-} u_{j}^{-} .
$$

The action of $S[\Phi]$ describing the dynamics and the couplings of the superfields $\Phi$ is similar to that of $2 \mathrm{~d}\left(\left(\frac{1}{2}\right)^{4}, 0\right) \mathrm{su}(2)$ harmonic superspace. More generally the matter couplings of the $\left.\left(\left(\frac{1}{3}\right)^{2},\left(\frac{1}{3}\right)^{2}\right)\right) \mathrm{su}(2)$ fractional supersymmetry, extending Eqs (44-45) by adjoining the antianalytic part, read in the harmonic superspace formulation [ 9 ] as:

$$
S[\Phi]=\int d^{2} z d^{2} \theta_{ \pm 1 / 3}^{+} d^{2} \theta_{\mp 2 / 3}^{+} d u L^{+4}(\Phi),
$$

where $L^{+4}$ is a functional of the superfield $\Phi$.

\section{Discussions and conclusion}

Using conformal parafermionic field theoretical methods, we have studied realisations of the fractional supersymmetric algebras extending the usual $2 \mathrm{~d}$ supersymmetries. We have found that all the known difficulties present in models based on $U_{q}(s l(2))$ and non commutative geometry representations are overpassed. One of the consequences of the new approach is that instead of one generator $Q_{-1 / k}$, FSAs are generated by many basic charge operators $Q_{-x}, \quad x=1 / k, 1 / 2 k, \ldots$, For $\mathrm{K}=3$ for example, the $\left(\frac{1}{3}, 0\right) \mathrm{FSA}$ is generated by two main charge operators $Q_{-1 / 3}$ and $Q_{-2 / 3}$ satisfying Eqs (33) and the $\left(\left(\frac{1}{3}\right)^{2}, 0\right)$ FSA is generated by two doublets $Q_{-1 / 3}^{ \pm}$and $Q_{-2 / 3}^{ \pm}$verifying Eqs (35). The proliferation of the fractional supersymmetric generators is the price one should pay in order to build a 2d local fractional supersymmetric QFT. This feature explains why the realisation obtained in ref [8] solves the constraints of fractional supersymmetry. It tells us moreover that there is a close connection between $\left(\frac{1}{3}, \frac{1}{3}\right)$ and $\left.\left(\left(\frac{1}{3}\right)^{2},\left(\frac{1}{3}\right)^{2}\right)\right)$ FSA's and $2 \mathrm{~d} \mathrm{~N}=2 \mathrm{U}(1)$ and $\mathrm{N}=4 \mathrm{su}(2)$ supersymmetric algebras respectively. On the other hand the conformal field methods show us that fractional supersymmetry has other remarkable features which become manifest for $K \geq 3$. As an example, the standard 
definition of fractional heterotic superalgebras as $Q^{K}=P ; \quad k>2$ is a formal one. The right way to define it is as $P=\operatorname{tr}\left[Q^{K}\right]$ where $\mathrm{Q}$ is a $K \times K$ matrix. For $\mathrm{K}=3$, we found that $Z_{3}$ invariant fractional supesymmetric algebra is generated by $Q_{-x}, \quad x=\frac{1}{3}, \frac{2}{3}, \ldots$ together with $Q_{0}^{ \pm}$rotating the components of these doublets. The energy momentum vector operator $P_{-1}$ in the $Z_{3} \mathrm{FSA}$ is just the trace of the following $3 \times 3$ matrix:

$$
\mathbf{Q}^{3}=Q_{0}^{+} Q_{-2 / 3}^{+} \Pi_{1}+Q_{-1 / 3}^{+} Q_{0}^{+} \Pi_{2}+Q_{-2 / 3}^{+} Q_{-1 / 3}^{+} \Pi_{3},
$$

where the $\Pi_{i}$ 's are projectors on the states $|i\rangle, \quad i=1,2,3$. This new result is different from the one used in the literature according to which $\mathbf{Q}^{3}=\mathbf{Q}_{-1 / 3} \mathbf{Q}_{-1 / 3} \mathbf{Q}_{-1 / 3}$ is a C-operator. Furthermore the method based on parafermions we have been using in this study tells us also that fractional spin objects may be treated as anticommuting objects. The point is that all fractional spin objects involved in the game are valued in the gl(3) nilpotent subalgebra and more precisely their square is identically zero; see Eqs (29). This anticommuting feature is reconfirmed by the braiding property of conformal fields which shows that the field operators $\Psi_{\Delta_{1}}$ and $\Psi_{\Delta_{2}}$ of fractional spin such that $\Delta_{1}+\Delta_{2}=1$, should anticommute. In particular the correlation function $\left\langle\Psi_{-1 / 3}\left(z_{1}\right), \Psi_{-2 / 3}\left(z_{2}\right)\right\rangle$ is equal to $-\left\langle\Psi_{-2 / 3}\left(z_{1}\right), \Psi_{-1 / 3}\left(z_{2}\right)\right\rangle$; a fact which imply that one may build a local $2 \mathrm{~d}$ quantum field theory invarint under fractional supersymmetry. In the end of discussion, we would like to note that as far as locality is concerned, the building of a local $2 \mathrm{~d}$ fractional supersymmetric theory may be achieved by linearizing the cubic relation $\mathrm{Q}^{3}=P_{-1}$. There are differents ways to perform this linearisation. A way to do it is as we have done in this study where Eqs (26) and (28) are remplaced by Eqs (35-39). Another way is as done in [8]where the nilpotency condition $\theta_{1 / 3}^{3}=0$ , $\theta_{1 / 3}^{2} \neq 0$, necessary for the construction of generalized superspace formalism is solved of two anticommuting Grassman variables $\theta_{1 / 3}^{+}$and $\theta_{2 / 3}^{-} ; \theta_{1 / 3}^{+2}=\theta_{2 / 3}^{-2}=0$. Though this linearisation works, no explanation was given in [8]. In the present analysis this feature can be derived in a rigourous way. the analogue of the higher order nilpotency condition $\theta_{1 / 3}^{3}=0, \theta_{1 / 3}^{2} \neq 0$ is trivially satisfied here since $\theta_{1 / 3}^{+2} \theta_{2 / 3}^{+}=\theta_{1 / 3}^{+} \theta_{1 / 3}^{+2}=0$.

\section{Aknowledgements}

The authors would like to thank Dr A.Elfallah and Dr J.Zerouaoui for stimulating discussions. This research work has been supported by the programm PARS under contrat 372-98 CNR. 


\section{References}

[1] Leclair A and Vafa C 1993 Nucl. Phys. B 401413

(Leclair A and Vafa C C 1992 Preprint CLNS 92/1150, HUTP 92/A045)

Fateev V and Zamolodchikov A B, Nucl. Phys. B 280644

Bais F, Bouwknecht P, Surridge M and Schoutens K 1988 Nucl. Phys. B 304348

Bais F, Bouwknecht P, Surridge M and Schoutens K 1988 Nucl. Phys. B 304371

Durand S, 1993 Phys. Lett B 312115

Durand S, 1993 Mod. Phys. Lett A 82323

[2] Bernard D and Leclair A 1990 Nucl. Phys. B340 721

C. Ahn, D. Bernard and A. Leclair, 1990 Nucl. Phys. B346409

Saidi E H, Sedra M B and Zerouaoui J, 1995 Class. Quantum Grav. 121567.

Mussardo G 1992 Phys Rep. 218216

[3] Perez A, Rausch de Traubenberg M and Simon P 1996 Nucl. Phys.B482325

( Perez A, Rausch de Traubenberg M and Simon P 1996 2D Fractional Supersymmetry for Rational Conformal Field Theory: Application for Third-Integer Spin States,preprint LPT-96-07, hep-th/9603139)

Kadiri A, Saidi E H, Sedra M B and Zerouaoui J, 1994 On exotic supersymmetries of the thermal deformation of minimal models preprint IC/94/216

[4] Elfallah A, Saidi E H and Zerouaoui J,1998 on finite dimentional fractional superalgebras Preprint UFR-HEP

[5] Zamolodchicov A B and Fateev V A , 1985 Sov. Phys. JETP62 215

Belavin A A, Polyakov A M, Zamolodchikov A B, 1984 Nucl. Phys. B214 333

[6] Freidan D, Qiu Z and Shenker S, 1985 Phys. Lett. B151 37

Bershadsky M A, Knizhnik V G and Teitelman M G, 1985 Phys. Lett. B15131

[7] Gepner D and Qiu Z, 1987 Nucl. Phys. B285423

Chakir H, Elfallah A and Saidi E H, 1995 Mod. Phys. Lett 382931

[8] Saidi E H, Sedra M B and Zerouaoui J , 1995 Class. Quantum. Grav.12 2705

[9] Sokatchev E and Stelle K S, 1987 Class. Quantum. Grav. 4501

Lhallabi T and Saidi E H 1988 Int. J. Mod. Phys. A 3187

[10] Elfallah A PhD Thesis LMPHE / 96 Kakushadze Z and Henry Tye S H, 1994 Phys Rev D49 4122

[11] Galperin A Ivanov E, Ogievetsky V and Sokatchev E 1985 Class. Quantum Grav.2 617 
[12] Zumino B 1979 Phys. Lett. B87 203 